Cahiers de recherches médiévales

\title{
Le débat sur le Roman de la Rose
}

comme document d'histoire littéraire et morale

\section{Virginie Greene}

\section{(2) OpenEdition \\ Journals}

Édition électronique

URL : https://journals.openedition.org/crm/2586

DOI : $10.4000 / \mathrm{crm} .2586$

ISSN : $1955-2424$

Éditeur

Honoré Champion

Édition imprimée

Date de publication : 30 juin 2007

Pagination : 297-311

ISSN : 1272-9752

Référence électronique

Virginie Greene, «Le débat sur le Roman de la Rose », Cahiers de recherches médiévales [En ligne], 14 spécial | 2007, mis en ligne le 30 juin 2010, consulté le 15 décembre 2022. URL : http://

journals.openedition.org/crm/2586; DOI : https://doi.org/10.4000/crm.2586 


\title{
RM
}

\author{
Le débat sur le Roman de la Rose \\ comme document d'histoire littéraire et morale
}

Le petit corpus de textes constituant ce que l'on convient d'appeler «le débat sur le Roman de la Rose» nous offre l'occasion d'écouter l'écho d'une discussion historique portant sur un sujet littéraire : la valeur esthétique et morale du Roman de la Rose. En même temps, la mise en textes (en lettres ou en traités) de la discussion est un processus littéraire qui ne manque pas d'intérêt en lui-même. Dans cet article je présenterai mon écoute et ma lecture d'une lettre de Christine de Pizan adressée à Jean de Montreuil ${ }^{160}$. La lettre répond à l'éloge du Roman de la Rose de Jean de Meun que Montreuil a écrit et fait circuler dans un petit traité aujourd'hui perdu. Prenant le contrepied de Montreuil, Christine compose une critique détaillée du même ouvrage ${ }^{161}$. Son texte peut donc bien être qualifié de lettre ou épître, puisqu'il répond précisément à un autre texte et s'adresse à son auteur. Mais par sa longueur, son sujet et sa structure argumentative, il relève également du traité en prose, un genre en plein développement en langue vernaculaire à cette époque et auquel Christine s'essaye précisément dans cette lettre.

La lettre, qui n'est pas datée, semble avoir été écrite pendant l'été $1401^{162}$. Nous n'en avons pas l'original et ne la connaissons que comme pièce d'un dossier comprenant d'autres lettres discutant le Roman de la Rose. Ce dossier est conservé dans huit manuscrits tous datés de la première moitié $d u X^{\mathrm{e}}$ siècle, c'est-à-dire tous assez proches de l'époque où ces lettres ont été écrites, entre le printemps 1401 et l'automne 1402. Tous ces dossiers ne sont pas identiques (certains sont plus complets que d'autres et les textes des lettres présentent des variantes) mais sept des huit manuscrits dérivent de dossiers constitués par Christine elle-même, et qu'elle a d'abord fait circuler indépendamment avant de les inclure dans une édition de ses œuvres. Le dossier du huitième manuscrit ( $\mathrm{BnF} 1563$ ) émane apparemment de l'autre camp (les rodophiles, comme les surnomme Éric Hicks), puisqu'il est accompagné du Roman de la Rose et d'autres textes de Jean de Meun, et qu'il comprend une lettre de Pierre Col répondant à Christine et défendant Jean de Meun et les rodophiles. Cette lettre ne figure pas dans les autres manuscrits ${ }^{163}$.

\footnotetext{
${ }^{160}$ Jean de Montreuil n'est jamais nommé ainsi dans les manuscrits, mais «Jean Johannès », «Jehan Charlin de Monstereul» ou par son titre de prévôt de Lille. Sur son identité et son nom, voir A. Thomas, «Le nom et la famille de Jehan de Monstereul », Romania, 37, 1908, p. 594-602.

${ }^{161}$ Les citations de la lettre de Christine viennent de : Christine de Pisan, Jean Gerson, Jean de Montreuil, Gontier et Pierre Col, Le Débat sur le Roman de la Rose, éd. É. Hicks, Paris, Champion, 1977, réimpr. Slatkine, 1996 (abrégé ci-après Débat). Sur le traité perdu de Montreuil, voir p. xv-xix.

${ }^{162}$ Le Débat, p. lii-liv.

${ }^{163}$ Sur ces manuscrits voir Débat, p. lv-xcii. Pour une bibliographie récente sur le débat, voir : Le Débat sur Le Roman de la Rose, tr. V. Greene, Paris, Champion, 2006, p. 313-336.
}

Cahiers de Recherches Médiévales (XII -XV s.), 14spé, 2007 
Les protagonistes de cet échange de vues entrent dans deux catégories : les plus connus et les moins connus. Les plus connus sont les rodophobes: Christine de Pizan, femme de lettres, et Jean Gerson, chancelier de l'Université de Paris ${ }^{164}$. Les rodophiles sont deux secrétaires du roi Charles VI, Jean de Montreuil et Gontier Col, et un chanoine de Notre-Dame de Paris, le frère de Gontier, Pierre Col. Le moins obscur des trois, Jean de Montreuil, a laissé une œuvre épistolaire en latin assez importante, qui lui a valu d'être considéré comme un pionnier de l'humanisme en France. Sa carrière professionnelle est assez bien connue grâce à ses lettres ${ }^{165}$. Des frères Col, on n'a conservé que les textes du dossier sur le Roman de la Rose et rien ne semble indiquer qu'ils aient produit une œuvre littéraire ou didactique. Jean de Montreuil et Gontier Col moururent en 1418, massacrés par les Bourguignons. On ne sait ni quand ni comment mourut Pierre $\mathrm{Col}^{166}$.

$\mathrm{Au}$ centre du débat, Le Roman de la Rose, roman allégorique de plus de 20000 vers, œuvre littéraire majeure, qui n'a pas été découverte au XIX ${ }^{\mathrm{e}}$ ou au XX siècle mais a été reconnue comme digne d'attention et de diffusion dès la fin du $\mathrm{XIII}^{\mathrm{e}}$ siècle. La quête de la Rose par l'Amant héros et narrateur du roman est la cause et le prétexte d'une extraordinaire prolifération de discours lyriques, didactiques, satiriques, parodiques, dialectiques, mythologiques, érotiques, voire, selon certains lecteurs, pornographiques. C'est une œuvre qui ne résout aucunement ses nombreuses contradictions et donc suscite des lectures contradictoires, ce qui semble une des raisons de son succès. Le débat sur Le Roman de la Rose a probablement commencé dès que le roman a circulé sous sa forme complète bicéphale ainsi qu'en témoignent les nombreux manuscrits comportant des annotations, illustrations, variantes, et des marques de censures ${ }^{167}$. Et ce débat continue encore aujourd' hui ${ }^{168}$.

\footnotetext{
${ }^{164}$ Sur l'abondante bibliographie des études pizaniennes, voir: A. J. Kennedy, Christine de Pizan : A Bibliographical Guide, Londres, Grant and Cuttler, 1984 ; et du même : Christine de Pizan: A Bibliographical Guide (supplement), Londres, Grant and Cuttler, 1994. Jean Gerson n'a pas bénéficié d'une telle attention, mais a profité d'un intérêt accru pour le moyen âge tardif et ses intellectuels. Voir par exemple: B. P. McGuire, Jean Gerson and the Last Medieval Reformation, University Park (PA), Pennsylvania State University Press, 2005.

${ }^{165}$ Jean de Montreuil, Opera, éd. E. Ornato, 4 vol., Turin, G. Giapichelli, 1963. Sur Montreuil et son milieu professionnel et littéraire, voir: E. Ornato, Jean Muret et ses amis Nicolas de Clamanges et Jean de Montreuil, Genève-Paris, Droz, 1969 ; G. Ouy, "Jean de Montreuil et l'introduction de l'écriture humanistique en France au début du $\mathrm{XV}^{\mathrm{e}}$ siècle » dans Litterce Textuales : Essays Presented to G.I. Lieftinck, éd. J. P. Gumbert et M. J. M. de Haan, 4 vol., Amsterdam, Van Gendt, 1972, vol. 4 : Miniatures, Scripts, Collections, p. 53-61.

${ }^{166}$ Sur les frères Col, voir: A. Coville, Gontier et Pierre Col et l'humanisme en France au temps de Charles VI, Paris, Droz, 1934, réimpr. Slatkine, 1977.

${ }^{167}$ Sur la réception médiévale du Roman, voir P. Y. Badel, Le Roman de la Rose au XIV siècle, Genève, Droz, 1980 et S. Huot, The "Romance of the Rose » and its Medieval Readers: Interpretation, Reception, Manuscript Transmission, Cambridge, Cambridge University Press, 1993, p. 22-27.

${ }^{168}$ Voir V. Greene, «Le débat et la conversation », introduction à : Le Débat sur Le Roman de la Rose, 2006, p. 7-38.
} 
En dépit de cette situation unique du Roman de la Rose dans la tradition littéraire française et en dépit de la position importante de certains des interlocuteurs, la discussion de 1401-1402 pour ou contre la Rose aurait pu rester une discussion privée, sans écho pour la postérité. La discussion est devenue débat et, comme telle, est entrée dans l'histoire littéraire française parce que certains des protagonistes (et en particulier Christine de Pizan) ont jugé que les idées échangées (et surtout les leurs) méritaient d'être conservées sous forme d'un texte à lire et à relire. Christine considère sa contribution écrite au débat comme une partie de son œuvre littéraire. De fait, ses Espitres sur le Rommant de la Rose (comme elle les nomme dans le Livre de la Cité des Dames ${ }^{169}$ ) marquent un point tournant dans sa carrière d'écrivain. En écrivant ces lettres, elle passe de la poésie lyrique courtoise qu'elle pratiquait dans un cercle choisi mais restreint, à la littérature didactique en vers ou en prose, touchant à des sujets tels que la morale, la politique, la philosophie, la science, la religion. Suivant le modèle littéraire proposé par Jean de Meun tout en l'attaquant, Christine revendique ainsi pour l'auteur en vernaculaire un statut comparable à celui attribué aux Auteurs - les auteurs écrivant en latin. Du même coup elle revendique la possibilité pour une femme (exclue a priori de la culture latine) d'être auteur quand même ${ }^{170}$. Si la discussion a porté surtout sur des questions de morale et d'esthétique telles que la position de l'auteur dans la cité et sa responsabilité morale, les enjeux du débat mis en écrit incluent la position des femmes dans la cité ainsi que le discours sur les femmes. On peut dire que, d'une certaine façon, Christine a détourné une discussion en cours pour en commencer une autre. Dans l'étude qui suit, j'étudierai la manière dont Christine rend compte de l'origine de la discussion, décrit sa position de lectrice du Roman de la Rose et définit sa conception du langage comme outil social autant que comme outil intellectuel. Enfin j'examinerai comment Christine relie le problème de l'injustice à l'égard des femmes aux questions de morale et d'esthétique qui servent de point de départ au débat².

\section{La genèse du débat}

Au début de sa lettre, Christine remercie Jean de Montreuil de lui avoir envoyé son traité dans une longue phrase qui lui permet de rappeler à Montreuil la genèse du débat, ses circonstances, et d'affirmer sa position sans ambiguïté ni ménagement :

Et comme il vous ait pleu de vostre bien, dont vous mercy, moy envoier un petit traictié ordenné par belle rethorique et voirsemblables raisons (lequel est de voz diz fait en reprenant, comme il me semble, aucuns blameurs de la compilacion du Rommant de la Rose en aucunes pars et moult soustenant ycellui et approuvant l'uevre et les aucteurs d'icelle et par especial Meun), je, ayant leu et considéré vostre dicte prose et compris l'effet selon la legiereté de mon petit engin - combien que a

${ }^{169}$ Débat, éd. É. Hicks, p. 193.

${ }^{170}$ Voir K. Brownlee, «Christine de Pizan : Gender and the New Vernacular Canon », Early Women Writers and Canons in England, France and Italy, éd. P. J. Benson et V. Kirkham, Ann Arbor (MI), University of Michigan Press, 2005, p. 99-120. 
moy ne soit adreçant ne response ne requiert, mais meue par oppinion contraire a voz dis et acordant a l'especial clerc subtil a qui vostre dicte espistre s'adrece -, vueil dire, divulguer et soustenir manifestement que, sauve vostre bonne grace, a grant tort et sans cause donnéz si perfaicte louenge a celle dicte euvre, qui mieulx puet estre appellee droicte oysiveté que œvre utile, a mon jugement (Débat, p. 12).

Christine présente ainsi les faits qui l'ont amenée à prendre la plume: d'«aucuns blameurs » ont critiqué Le Roman de la Rose; Montreuil a écrit un traité prenant la défense du Roman et l'a adressé à l'un d'eux, un certain «clerc subtil » et à Christine; Christine, qui est d'accord avec le clerc subtil, décide de répondre à Montreuil. Ce qui n'est pas très clair, c'est à quel moment et comment Christine est entrée dans le débat. Est-ce qu'elle faisait partie du groupe des blâmeurs du début? Est-ce qu'elle est proche du «clerc subtil» qui l'aurait appelée à la rescousse une fois que Montreuil aurait affirmé sa position?

Il existe deux autres versions du début de la discussion. Christine commence en ces termes un petit récapitulatif qui accompagne les pièces du dossier qu'elle a fait rassembler :

Comme ja pieça paroles feussent meues entre mon seigneur le prevost de Lisle, maistre Jehan Johannes, et Cristine de Pizan touchans traictiéz et livres de pluseurs matieres, esquelles dictes paroles le dit prevost ramentut Le Rommant de la Rose en lui actribuant tres grant et singuliere louenge et grant dignité [...] (Débat, p. 8).

Cette fois ce serait à la suite d'une conversation entre Christine et Montreuil que celui-ci aurait envoyé une épître défendant le Roman de la Rose à un clerc de ses amis qui était d'accord avec Christine, ainsi qu'à Christine elle-même. La troisième version se trouve dans une lettre en latin adressée par Montreuil à un ecclésiastique important (peut-être Pierre d'Ailly). Se trouvant dans une période de loisir et n'ayant pas grand chose à faire à la chancellerie, il a enfin lu Le Roman de la Rose, que son ami Gontier Col lui recommandait avec insistance, et dans l'enthousiasme de sa lecture, il a rédigé un traité en français faisant l'éloge du génie de l'auteur (actoris ingenium). Il envoie ce traité au révérend père à qui il écrit pour savoir ce qu'il en pense (Débat, p. 28). Dans cette lettre à une autorité supérieure, Montreuil ne fait aucune allusion à une discussion sur le Roman de la Rose avec un autre clerc et une femme, et décrit son traité gallica scriptione comme une distraction et un exercice rhétorique.

La version la plus vraie devrait logiquement être celle de la lettre de Christine à Montreuil puisque ce n'est pas un récit à un tiers, mais un rappel de ce que son interlocuteur doit fort bien savoir. Dans ce cas, dans son résumé accompagnant le dossier et écrit plusieurs mois après la lettre à Jean de Montreuil ${ }^{171}$, Christine arrange quelque peu le récit du début de la querelle pour se poser en première protagoniste de Jean de Montreuil et en championne du clan des rodophobes. Cet arrangement

${ }^{171}$ Éric Hicks date la lettre de Christine à Montreuil de juin ou juillet 1401, alors que la lettre par laquelle Christine adresse le dossier à la reine Isabeau porte la date du $1^{\text {er }}$ février 1402 . Débat, p. lii et p. 6. 
des faits en récit marque le moment où la conversation mondaine qui a commencé d'une manière un peu floue entre elle, Montreuil et un clerc ami de Montreuil mais d'opinion opposée à lui, se transforme en échange de lettres, exercice d'écriture, polémique, argumentation, plaidoyer. La mise en écrit donne du poids aux mots et opinions échangés et fixe l'histoire du débat. Christine annonce aussi qu'elle prend cette discussion au sérieux et qu'elle entend en faire un débat public, alors qu'apparemment Jean de Montreuil aurait préféré garder ce débat à l'intérieur d'un petit cercle de lettrés et si possible ne pas y inclure Christine.

$\mathrm{Au}$ début de sa lettre à Montreuil, Christine indique le ton et la stratégie qu'elle va utiliser tout au long du débat. Elle commence avec les formules traditionnelles d'hyperbole à propos du destinataire («tres chier sire et maistre, saige en meurs, ameur de science, en clergie fondé et expert de rethorique », p. 11-12) et de dénigrement de soi-même ( «femme ignorant d'entendement et de sentement legier», p. 12) qui prouvent qu'elle connaît les usages littéraires et épistoliers, en bref qu'elle sait elle aussi écrire une lettre. Mais le miel du début rend le vinaigre de la suite d'autant plus acide, comme l'indique la formule «sauve vostre bonne grace » qui annonce toujours une pique, en l'occurrence « a grant tort et sans cause donnéz si perfaicte louenge a celle dicte euvre»(p.12). Christine déclare ainsi que la réputation et la qualité intellectuelle de son interlocuteur ne l'impressionnent pas et ne l'empêcheront pas d'attaquer vigoureusement l'opinion qu'il défend. La métaphore militaire de l'assaut et de la défense joue un rôle clé aussi bien dans le Roman de la Rose que dans le débat ${ }^{172}$. Christine ne l'utilise pas directement, mais se place explicitement en position d'attaquante, refusant le rôle traditionnel de la femme place-forte à prendre et qui doit se défendre contre l'assaut masculin. Ce faisant, elle impose la position féminine à Montreuil, ce qui explique peut-être pourquoi Montreuil n'a pas lui-même répondu à sa lettre et a déféré la tâche de défendre Jean de Meun à deux amis, les frères Col, que Christine n'avait pas provoqués et qui pouvaient donc adopter en face d'elle une position moins personnellement défensive. Il est aussi à remarquer que quand Jean Gerson fait allusion à Christine dans une lettre en latin à Pierre Col, il la désigne comme virilis illa femina (Débat, p. 168), terme élogieux mais sur lequel Gerson ne s'appesantit pas. Dans les écrits de Gerson associés au débat, on trouve peu d'allusions à son «alliée» alors que Christine en fait beaucoup plus à lui dans sa lettre à Pierre Col (Débat, p. 118-121).

Le problème majeur de Christine n'est pas de débattre (ni même de débattre contre des adversaires plus habitués à ce genre de jeu qu'elle) mais d'entrer en débat et de s'assurer qu'un débat avec ou contre elle sera pris au sérieux. Le flottement entre les différentes versions de la genèse du débat ainsi que l'évidente dérobade de Jean de Montreuil indiquent que le passage de la discussion mondaine au débat écrit permet à Christine d'aborder ce qui est pour elle le fond de la question Jean de Meun - la misogynie dans les lettres - d'une manière sérieuse, enfin. Le sérieux de Christine, qui s'oppose à l'esprit plus ludique des lettres de ses adversaires, ne l'empêche pas de faire usage d'humour et d'ironie, voire de reconnaître que son

\footnotetext{
${ }^{172}$ Guillaume de Lorris et Jean de Meun, Le Roman de la Rose, éd. et tr. A. Strubel, Paris, Livre de Poche, 1992, v. 15107-15138 et Débat, p. 104-106, 128, 136-137.
} 
adversaire tient à son opinion autant qu'elle tient à la sienne ${ }^{173}$. Mais ce sérieux assumé dans l'acte d'écrire publiquement à Jean de Montreuil se reflète dans la position de Christine vis-à-vis de la littérature et de la lecture d'œuvres littéraires. La littérature est pour elle une affaire sérieuse et qui doit s'assumer clairement et publiquement. On a pu reprocher à Christine une position moralisante et raide, ne laissant pas place au jeu ni à l'ambiguïté, ne distinguant pas entre réalité et représentation, aboutissant logiquement (sinon historiquement) à la censure et à la répression ${ }^{174}$, et ce reproche trouve ample justification dans la lettre à Montreuil. Mais si l'on veut comprendre le débat dans toutes ses dimensions, il convient sans doute d'examiner davantage le sérieux de Christine. Du point de vue de Christine, en son temps et dans la société qui est son milieu, il faut que la littérature soit matière sérieuse pour valoir la peine qu'une femme s'y engage. Qu'une femme se rende maître d'un art que l'on puisse qualifier de frivole, sans portée réelle, ne peut que renforcer la réputation de frivolité du sexe féminin. L'effort de Christine pour devenir un maitre des lettres est trop réel pour s'accommoder d'ironie et d'ambiguïté au niveau fondamental où l'auteur s'identifie à ce qu'elle écrit. Voilà pourquoi la première accusation portée par Christine contre l'œuvre de Jean de Meun est que celle-ci «mieulx puet estre appellee droicte oysiveté que œvre utile, a mon jugement» (Débat, p. 12). En faisant l'éloge d'une œuvre qui n'est qu'oisiveté, Montreuil a lui aussi fait œuvre d'oisiveté. En ancien et moyen français «oysiveté » peut signifier l'oisiveté au sens moderne, mais aussi la paresse, la lâcheté et la sottise. C'est aussi le nom d'une des allégories du Roman de la Rose. Dans la partie du roman de Guillaume de Lorris, Oiseuse, concierge du jardin de Déduit, ouvre la porte à l'Amant (v. 525-584). L'oisiveté est mère de tous les vices et de tous les raffinements. Elle est aussi ce qui enferme la femme dans un jardin où elle n'a rien d'autre à faire qu'à se laisser cueillir. En écrivant contre l'œuvre de «droicte oysiveté » et ceux qui en font l'éloge, Christine fonde la genèse de son geste d'écrivain dans l'imaginaire (il n'est pas sûr qu'elle ait été dès le début la championne qu'elle devient par la suite) et dans l'obsession de faire œuvre utile. Mais est-ce qu'en lisant l'œuvre d'oisiveté dont elle veut débattre, Christine n'a pas basculé elle-même dans la paresse, la lâcheté et la sottise ? Si l'œuvre littéraire est prise sérieusement, sa lecture a des implications sérieuses et l'exercice critique prend une autre portée que celle de l'échange de goûts et d'opinions dans la conversation mondaine.

\footnotetext{
${ }^{173}$ Voir la fin de sa lettre à Pierre Col, Débat, p. 145-150, où elle dit entre autres : « Je ne say a quoy tant nous debatons ces questions, car je croy que ne toy ne moy n'avons talent de mouvoir nos oppinions : tu dis qu'il est bon ; je dis qu'il est mauvais » (p. 145). Ce qui n'est pas une position relativiste, puisque Christine affirme jusqu'à la fin de sa lettre son sentiment d'avoir raison, mais une reconnaissance des positions respectives, ce qui implique une certaine distance vis-à-vis du débat.

${ }^{174}$ Voir W. Calin, «Is Jean de Meun antifeminist?», «Riens ne m'est seur que la chose incertaine » : Études sur l'art d'écrire au Moyen Âge offertes à Eric Hicks, Genève, Slatkine, 2001, p. 81-90, et D. Hult, «Words and Deeds : Jean de Meun's Romance of the Rose and the Hermeneutics of Censorship », New Literary History, t. 28, 2, 1997, p. 345-366.
} 


\title{
Lire le Roman de la Rose
}

Un peu plus loin dans sa lettre à Montreuil, Christine développe et précise l'accusation d'oisiveté :

\begin{abstract}
Mais pour quoy ay je devant dit que «mieulx puet estre appellee oysiveté... »? Sans faille, il me semble que toute chose sens preu, non obstant soit traittee, faicte et accomplie a grant labeur et paine, puet estre appellee oyseuse ou pis que oyseuse de tant come plus mal en ensuit (Débat, p. 13).
\end{abstract}

En revenant d'elle-même sur les termes de l'accusation qu'elle vient de porter quelques lignes plus haut, Christine indique qu'elle n'écrit pas à la légère, qu'elle pèse ses mots, et que la légèreté elle-même (impliquée dans l'idée d'oisiveté) ne dois pas se traiter légèrement. L'accusation d'oisiveté est grave, explique Christine, car elle implique que l'effort et le travail qu'une telle œuvre a pu réclamer sont annulés par le manque de profit résultant de l'œuvre. Autrement dit, l'auteur ne peut ni se flatter ni s'excuser de rien en arguant de son temps de travail ou de sa maîtrise technique. L'œuvre doit être jugée à la mesure du bien ou du mal qui en découlent. Une œuvre « oyseuse » est une œuvre qui ne produit aucun bien. Une œuvre «pis que oyseuse » est une œuvre qui produit du mal. Dans les deux cas, le travail et l'effort d'écrire, autant que le travail et l'effort de lire, sont annulés ou rendus négatifs. Ce qui ne signifie pas que Christine rejette le travail au seul profit du cœur pur et de la bonne intention. Son œuvre (à commencer par cette lettre) en témoigne. Il faut non seulement que l'écrivain travaille à posséder son métier, mais aussi qu'il travaille de telle sorte à ce que son œuvre produise du bien. Grande, belle et dangereuse exigence.

Quel bien et quel mal peuvent résulter ou être produits par un roman? Seul le lecteur peut le dire, car si la valeur du livre ne peut se mesurer en fonction de l'effort fourni par l'auteur, elle doit se mesurer en fonction de l'impact produit dans l'esprit du lecteur au terme de la lecture. Christine poursuit donc son explication en décrivant son expérience de lectrice du Roman de la Rose :

Et comme ja pieça pour la grant renommee commune du dit rommans desiray le veoir, aprés que congnoissance m'ot un petit fait entendre choses subtiles, le leu et consideray au long et au lé le mieulx que le sceu comprendre. Vray est que pour la matiere qui en aucunes pars n'estoit a ma plaisance m'en passoye oultre comme coc sur brese : si ne l'ay plenté veu (Débat, p. 13).

Christine a lu Le Roman de la Rose parce qu'elle en a entendu parler, ce qui est bien proche de nos expériences de lecture ${ }^{175}$. Mais elle ajoute qu'elle ne l'a lu qu'après avoir acquis les connaissances nécessaires pour comprendre des textes difficiles. Christine ne veut pas dire qu'elle s'est mise à étudier spécialement pour pouvoir lire Le Roman de la Rose, mais qu'elle n'a pu lire Le Roman de la Rose que parce qu'elle avait suffisamment lu d'autres textes «subtils» pour comprendre celui-là. Au-delà du désir de réaffirmer sa compétence à débattre avec des hommes de lettres, Christine reconnaît la nature complexe du Roman de la Rose, un livre qui reste

${ }^{175}$ Voir de Nathalie Sarraute, Les fruits d'or, (1963), roman de la renommée d'un roman. 
difficile à lire aujourd'hui comme naguère parce qu'il est rempli de références littéraires et philosophiques et parce qu'il est fait d'arguments complexes, mis en scène de manière complexe. Le livre que Christine a qualifié d' "oisif », est aussi un livre «subtil ». Ce n'est pas un livre idiot qui ferait perdre du temps par manque de substance et qui ne produirait aucun bien par défaut de subtilité. Si le livre est à la fois oisif et subtil, on peut s'attendre à ce qu'il penche vers la catégorie des œuvres «pis que oyseuses[s]», celles qui produisent du mal. Et le premier mal qu'a produit ce livre sur cette lectrice, cela a été de la troubler au point de rendre l'expérience de lecture à la fois fascinante et honteuse. Christine dit en effet à propos de sa lecture deux choses contradictoires: qu'elle l'a lu «au long et au lé au mieux que le sceu comprendre» mais aussi qu'elle en a sauté des passages «comme coc sur brese». Elle a tout lu avec bien du mal, mais elle n'a pas tout lu parce que cela lui faisait mal, ou du mal. On peut sourire de la contorsion de Christine, tout en appréciant sa description assez juste de ce qui arrive à un lecteur rencontrant un passage troublant (ce qui trouble varie selon le lecteur, son âge et son époque, mais tout lecteur peut rencontrer un passage troublant), le lisant sans le lire, y repensant avec gêne mais souvent. De plus, Christine étant femme, voire dame, pour elle clamer qu'elle aurait lu tout le Roman de la Rose sans trouble serait risquer de se disqualifier en tant que personne morale. Qu'une femme prétende en savoir assez pour comprendre « au lonc et au lé » le Roman de la Rose est un défi intellectuel lancé à une élite masculine ; qu'une femme prétende en savoir assez pour n'être à aucun moment choquée dans sa lecture est un aveu de vulgarité aux yeux de toutes et de tous. La femme ne peut ni apprécier ni manipuler l'obscénité sans devenir elle-même obscène. Christine s'en tire du mieux qu'elle peut en utilisant l'image du coq sautillant sur les braises, se brûlant un peu les pattes pour échapper à de plus graves brûlures. Mais qu'allait-il donc faire sur les braises, ce coq?

Pour préserver son honneur de femme et de dame tout en affirmant sa compétence de lectrice du Roman de la Rose, Christine ajoute :

\footnotetext{
Neantmoins demoura en ma memoire aucunes choses traictees en lui que mon jugement condempna moult et encore ne puet approuver pour contraire louenge d'autre gent. Bien est vray que mon petit entendement y considere grant joliveté, en aucunes pars, tres sollennellement parler de ce qu'il veult dire - et par moult beaulx termes et vers gracieux bien leonimes : ne mieulx ne pourroit estre dit plus soubtilment ne par plus mesuréz trais de ce que il voult traictier (Débat, p. 13).
}

Aux yeux de Christine la lecture sautillante et sélective n'empêche pas plus l'exercice du jugement que la qualité esthétique du poème n'excuse sa propension à causer du trouble chez un lecteur ou une lectrice de bonnes mours. La lecture opère sur plusieurs plans impliquant la mémoire qui retient à la fois le texte lu et l'impression produite par la lecture, le jugement qui fait de la lecture un acte personnel, l'entendement qui restitue à ce qui a été lu sa dimension d'écrit. En lisant le Roman de la Rose, Christine a enregistré ses émotions de lectrice, construit son opinion d'individu autonome et analysé le travail d'écriture de l'auteur. Cette description de la lecture du Roman de la Rose constitue la synthèse de la position subjective que Christine entend occuper sur la scène des lettres en tant que femme, individu et clerc, c'est-à-dire, en tant que professionnelle de la lecture et de 
l'écriture. Critiquer le Roman de la Rose est pour elle faire la démonstration qu'une femme peut avoir un accès personnel et professionnel au langage et à la littérature sans en être abîmée, que le coq peut marcher sur les braises sans être consumé, à condition qu'il garde conscience de la nature brûlante du langage.

\section{Les noms des choses}

La première critique spécifique de Christine sur le texte de Jean de Meun porte sur un passage du discours de Raison à l'Amant :

Mais en accordant a l'oppinion a laquelle contrediséz, sans faille a mon avis, trop traicte deshonnestment en aucunes pars - et mesmement ou personnage que il claime Raison, laquelle nommes les secréz membres plainement par nom (p. 13).

Raison nomme les «secréz membres » par leur nom commun d'abord en racontant à l'Amant l'histoire de la castration de Saturne par Jupiter :

\section{Joustice qui jadis regnot}

Au tens que saturne regne ot,

Cui jupiter coupa les coilles

Ausi com se fussent andoilles (v. 5531-5534).

Si Raison s'en était tenue là, il est probable que Christine n'aurait pas pris la peine de s'y arrêter. Mais Jean de Meun fait en sorte que l'écart provocateur de langage n'échappe à aucun lecteur en y revenant deux mille vers plus loin quand l'Amant (qui apparemment a l'esprit d'escalier) dit à Raison

Si ne vous tieng pas a cortoise

Quant vous m’avez coilles nommées (v. 6924-6925).

Malicieusement, Jean de Meun indique que l'accusation de discourtoisie est ellemême discourtoise (toi aussi tu l'as dit !) mais surtout que la question de politesse en dissimule une autre plus importante à ses yeux et que Raison va développer dans un passage mémorable du Roman:

Je te di devant dieu qui m'ot

Que je, quant mis les nons as choses,

que si reprendre et blasmer m'oses,

Coilles reliques apelasse

Et reliques coilles nomasse,

Tu, qui si m'en mors et depiques,

me redeïsse de reliques

que ce fust laiz mos et vilains.

Coilles est biaus non et si l'ains.

Si sont par foi coillons et vit,

Ains nus plus biaus gaires ne vit

Et quant pour reliques m'oïsses

Coilles nommer, le mot prisses 
Pour si bel et tant le prisasses

Que partout coilles aorasses

Et les baisasses en eglises

En or et en argent assises (v. 7104-7120).

Avec l'efficacité d'un exhibitionniste exposant les parties de son corps précisément nommées ici, Meun expose d'un seul coup l'arbitraire du langage et la double nature sexuelle et religieuse des fétiches. C'est pourquoi l'exposition est relativement brève: Raison pour une fois ne développe pas longuement son thème. Mais le flash verbal (dont on peut s'offusquer, ricaner ou que l'on peut trouver génial) brillant comme braise n'a pas échappé à Christine qui prend le relais de l'Amant choqué et poursuit à sa place la discussion proposée par Meun. À l'argument de Raison que les parties sexuelles ayant été créées par Dieu ne sont pas honteuses, et que par conséquent leurs noms ne devraient pas être sujet de honte, Christine oppose d'abord l'argument théologique de la chute : « es choses que Dieu a faictes n'a nule laidure et par conséquent n'en doit le nom estre eschivé, [...] ; mais par la pollucion de pechié devint homme inmonde»(p.13). Avant la chute, ni les noms ni les choses n'étaient honteuses ; mais depuis que l'homme a perdu son innocence, la honte hante les mots et les choses.

En opposant à la subtilité philosophique de Meun l'orthodoxie de la chute sur laquelle repose la subjectivité chrétienne, Christine indique qu'un tel langage ne peut être tenu que dans l'espace abstrait où l'allégorie prend voix et corps. Dans la cité humaine, l'homme ou la femme utilisant ce langage signifierait toute autre chose que ce que Meun veut et peut faire signifier à Raison. La preuve en est que Montreuil, aussi subtil soit-il lui-même, se trompe sur la valeur du langage quand, dans son éloge du Roman, il en appelle à l'autorité de la parole du Christ :

Encore proposez que Jhesu Crist, «en parlant des pecheresses, les appella meretrix », etc. Et que il les appellast par celui nom vous puis souldre que cellui nom de meretrix n'est mie deshonneste a nommer selon la vilté de la chose - et plus vilment pourroit estre dit mesmes en latin (p. 14).

Si Jésus a parlé de prostituées, il n'a pas parlé de putes. Un clerc expert en langues et langage devrait bien saisir une distinction de registre assez évidente pour qu'une femme la comprenne «mesmes en latin». Christine a trouvé la faille majeure de la stratégie défensive de Montreuil. En essayant de ramener le texte de Meun au niveau de l'anthropologie concrète plutôt que de le défendre là où il opère le plus efficacement, au niveau de l'anthropologie métaphysique, Montreuil prête le flanc à une attaque du type «vous ne pratiquez guère ce que vous prêchez ». Dans sa lettre répondant à Christine, Pierre Col défend avec vigueur la liberté de parole, soutient que la sexualité est fondamentalement innocente, se moque de la pruderie des femmes, mais il n'utilise pas une seule fois lui-même les mots incriminés ${ }^{176}$. Comme Christine, il parle des «membres secrez», ce qu'elle ne manquera pas de lui faire remarquer en lui écrivant:

${ }^{176}$ Débat, p. 89-91. 


\begin{abstract}
Mais tu, qui tant te debas et par tant de repliques que plainnement se doivent nonmer par nom et que bien dist la Raison Jehan de Meung, je te prie chierement - tu qui es son tres especial desciple, come tu dis - pour quoy ne les nomme plainnement en ton escripture sans aler entour le pot? Il me samble que tu n'es pas bon escolier car tu n'en suis pas bien la doctrine de ton maistre (p. 123).
\end{abstract}

La doctrine du franc-parler peut être défendue rationnellement mais elle est difficile à appliquer dans les rapports sociaux ordinaires. Si Jean de Meun avait écrit une lettre à une dame pour défendre le langage de sa Raison, aurait-il usé du même langage que dans la fiction de son roman?

Dans le Roman de la Rose, Nature et son confesseur Genius célèbrent la sexualité comme expression naturelle de la vitalité créatrice des espèces s'opposant à l'œuvre de la mort, mais aucun des deux n'utilise les termes préconisés par Raison, comme si, pour Jean de Meun, le pur obscène était du ressort de la pure raison. Quand on passe à des allégories mixtes comme celles de Nature et Genius, Nature forge des bébés à tour de bras (v. 16009-16016) tandis que Genius incite les seigneurs qui l'écoutent, à faire bon usage de la charrue, outil du paysan, et de la plume, outil du clerc (v. 19547-19737). À cette poétique et métaphysique de la sexualité située dans une utopie sociale où la honte n'a pas de place et où tous les outils se valent, Christine répond que la honte est naturelle à l'homme social :

Et que honte doit estre deboutee en parlant en publique des choses dont nature mesmes se hontoye, je dis que, sauve la reverence de l'autteur et la vostre, grant tort commectéz contre la noble vertu de honte, qui de sa nature reffraint les goliardises et deshonnestetés en dis et en fais ; et que ce soit grant vice et hors ordre de pollicie honneste et de bonnes meurs appert en mains lieux de l'Escripture saincte (p. 14).

La honte et la pudeur font partie de la nature humaine résultant de la chute. Et cette nature est éminemment sociale, ce qui est reconnu dans la Bible dès que Caïn, le fondateur des villes, assassine Abel, l'agriculteur. Christine rappelle la dimension anthropologique de la Bible pour contredire l'argument maladroit de Montreuil sur le franc parler du Christ. Ce faisant elle impose aux auteurs de suivre un code de morale littéraire conforme aux usages sociaux, aux conventions de décence et de pudeur de la société dans laquelle ils vivent. Ou sinon l'œuvre littéraire sera «droite oisiveté » ou pire. L'argument de Christine risque alors de contredire son projet d'auteur, car si elle-même pratiquait à la lettre ce qu'elle prêche, elle s'interdirait d'aller contre la coutume sociale qui limite le domaine des femmes à ce que les hommes veulent bien leur accorder et non à ce qu'une femme comme Christine peut vouloir accomplir. Elle ne peut énoncer la norme sans la contredire, puisqu'elle est supposée simplement y obéir. Elle ne peut d'autre part, comme on l'a vu plus haut, reprendre à son compte la position provocatrice et créatrice de Jean de Meun sans basculer dans une disqualification morale complète. Le rapport de Christine à la vérité et au franc-parler - rapport qui traditionnellement définit la position morale de l'auteur qui désire assumer une position morale - est perverti dès le départ par le fait qu'elle soit femme. Mais si la vérité se dérobe, la justice se révèle être un terrain plus solide pour établir une autorité intellectuelle et morale féminine. Le reproche 
capital que Christine fait à Jean de Meun dans sa lettre à Montreuil, ce n'est pas d'avoir été immoral mais d'avoir été injuste à l'égard des femmes.

\section{Médire des femmes}

Dans la première partie de sa lettre, Christine juge du texte littéraire à l'aune de l'anthropologie morale, soulignant d'abord son utopisme linguistique puis l'affectation de cynisme exprimée dans les discours de Raison, de la Vieille, du Jaloux et de Genius (p. 14-16). Le défaut d'une telle stratégie critique est de dénier toute spécificité à la fiction littéraire et à son pouvoir de dire le vrai à sa manière. Mais la force de l'argument réside dans sa capacité à saisir le trouble provoqué par le texte dans une autre dimension qui intègre la prétention esthétique de l'auteur. $\mathrm{Si}$ la multiplicité des voix et des perspectives, l'ambiguïté des discours, la contradiction entre les différents discours constituent l'esthétique du Roman de la Rose de Jean de Meun et son rapport singulier à la vérité, l'obsession misogyne qui traverse ces voix et ces discours ne peut pas être justifiée dans une perspective esthétique ni comme accès particulier à la vérité, ou comme le dit plus joliment Christine :

Et encore pour Dieu !... regardons oultre un petit : en quel maniere puet estre vallable et a bonne fin ce que tant et si excessivement, impettueusement et tres nonveritablement il accuse, blame et diffame femmes de pluseurs tres grans vices et leurs meurs temoingne estre plains de toute perversité ; et par tant de repliques et auques en tous personnages ne s'en puet saouler (p. 16-17).

«Il» renvoie sans ambiguïté à Jean de Meun, mentionné au paragraphe précédent. La récurrence de la diffamation des femmes est lue comme une signature de l'auteur, ce qui permet à Christine de répondre ensuite à un argument utilisé par les défenseurs de Jean de Meun : ce que disent les personnages ne doit pas être attribué à l'auteur. Ainsi quand le Jaloux part dans une diatribe contre les femmes se concluant sur « Toutes estes, serez ou fustes / De fait ou de volenté, putes» (v. 915960) ces propos excessifs doivent être considérés comme représentant la passion maladive du personnage et non comme la pensée ou l'opinion de l'auteur. Christine répond : «Car se dire me vouléz que ce face le Jaloux comme passionné, je ne scay entendre qu'il appartiengne a l'office de Genius...» (p. 17). Elle pointe ainsi une des difficultés du texte de Meun : le statut des différents personnages supports de discours, ou la fonction des différents modes d'allégorisation. On pourrait distinguer ainsi une allégorisation psychologisante des passions humaines dans les personnages du Jaloux, de la Vieille, d'Ami et de Bel Accueil et une allégorisation philosophique, incarnant des principes universels tels que Raison, Nature et Genius. Amour, comme dans la tradition platonicienne du Banquet, tient des deux. Christine joue ici sur la double prétention du texte de Meun, d'être à la fois œuvre littéraire (roman mettant en scène les passions humaines) et œuvre philosophique (traité de la nature et de la nature humaine). $\mathrm{Si}$, à la rigueur, on peut admettre une représentation de la misogynie comme élément d'une psychologie des passions, comment l'admettre dans un discours sur la nature des êtres et des choses ? Bien entendu, tant que les femmes ne se mêlent pas de philosophie et de principes, la naturalisation de la misogynie ne dérange personne. Christine signale ici que l'injustice la plus 
profonde est celle qui ignore toute une catégorie de personnes et qui soustrait cette catégorie à l'application de la justice. Il n'y a pas de mal à diffamer les femmes tant qu'elles ne sont pas «diffamables». Un des effets proprement révolutionnaires de la lettre de Christine à Montreuil est de rendre la femme diffamable, et du coup d'en faire un sujet de droit.

Christine souligne ensuite la contradiction flagrante qui éclate dans un discours à la fois misogyne et promoteur de l'hétérosexualité. En effet Genius, paraphrasant un vers de Virgile ${ }^{177}$ déclare :

Seigneur gardez vous de vos fames,

Se vos cors amez ne vos ames [...]

Fuiez, fuiez, fuiez, fuiez,

Fuiez, enfanz, fuiez tel beste [...]

Car tant est venimeuse beste

Par cors et par keue et par teste

Que, se de li vous aprouchiez

Touz vous troverez entouchiez;

Qu'il mort et point en traison

Quanqu'il ataint, sanz guerison.

Car de cestui venim l'ardure

Nus triacle ne la cure :

Riens n'i vault herbes ne racine ;

Seul fouir en est medicine.

Si ne di je pas toute voie

N'ainc ne fu l'entencion moie

Que les fames chieres n' aiez

$\mathrm{Ne}$ que si fuir les doiez

Que bien avoec euls ne gisiez (v. 16581-16625).

Fuir en couchant? Coucher en fuyant? Comme le dit Christine, il y a ici «malement grant contradiction de commander a fuir ce que il veult que on suive et suivir ce que il veult que on fuie» (p. 17). Le discours des principes (l'homme est...) se heurte ici de plein fouet au discours satirique (les femmes sont...). Christine revient à un autre discours misogyne tout aussi injuste mais plus cohérent: «Mais puis que tant sont perverses, ne les deust commander approuchier aucunement ; car qui inconvenient redoubte, eschiver le doit » (p. 17). Si la femme est perverse par nature, l'homme qui a souci de son salut doit éviter tout contact avec la femme, y compris le contact sexuel, recommandation que l'on trouve, par exemple, à la fin de l'Art d'aimer d'André le Chapelain. Le mélange de misogynie cléricale et de promotion de l'hétérosexualité que l'on trouve dans le Roman de la Rose est facile à attaquer car elle repose sur un syllogisme faux. La perversité est à fuir; les femmes sont

\footnotetext{
${ }^{177}$ Qui legitis flores et humi nascentia fraga, / frigidus, o pueri (fugite hinc!), latet anguis in herba (Vous qui cueillez des fleurs et des fraises nées de la terre, enfants ! fuyez: un serpent froid se cache dans l'herbe) (Bucoliques, III, v. 92-93). Ce vers n'a rien de misogyne : le serpent semble faire allusion à la mort qui peut frapper de jeunes êtres. Si c'est du désir amoureux qu'il s'agit, rien ne précise s'il s'agit d'un désir hétérosexuel ou homosexuel.
} 
perverses; il faut coucher avec les femmes. Plus difficile à combattre est le syllogisme logiquement juste et moralement injuste : la perversité est à fuir ; les femmes sont perverses; il faut fuir les femmes. C'est à démontrer la fausseté de la mineure de ce syllogisme que Christine consacrera son Livre de la Cité des Dames.

En conclusion de sa lettre, Christine revient à la question de la valeur de l'œuvre littéraire : qu'est-ce qui rend un livre digne d'éloge et qu'est-ce qui rend une œuvre utile ? Rassemblant les fils de son argument, elle reconnaît de nouveau à Jean de Meun du talent et de l'intelligence, mais cette reconnaissance de la qualité du maître ne l'empêche pas d'exercer son esprit critique à son égard et à l'égard de son ouvre :

Non obstant que mon jugement confesse maistre Jehan de Meun moult grant clerc soubtil et bien parlant, et trop meilleur œvre plus prouffitable et de sentement plus hault eust sceu mectre sus s'il s'i feust appliquié - dont fu domage —, mais je suppose que la grant charnalité, puet estre, dont il fu remply, le fist plus habonder a voulenté que a bien prouffitable, comme par les operacions communement sont congneues les inclinacions. Non obstant ce, je ne reppreuve mie Le Rommant de la Rose en toutes pars, car il y a de bonnes choses et bien dictes sans faille (p. 20-21).

Les deux «non obstant» et la rupture de construction marquée par le «mais» marquent l'oscillation du jugement, qui ne veut ni condamner injustement ni se laisser influencer par l'autorité ou la réputation. C'est aussi un jugement qui évite la généralisation : il s'agit de juger de Jean de Meun et non de tous les hommes ou de tous les clercs. "La grant charnalité » que Christine lui attribue est un trait individuel, ce qui indique que pour elle les «inclinacions» personnelles, en particulier sexuelles sont transposées directement dans l'œuvre littéraire. Nonobstant, Christine peut encore trouver de la qualité dans l'œuvre d'un auteur «de grand charnalité ». Mais cette qualité même rend l'œuvre d'autant plus dangereuse : «plus est adjoustee foy au mal de tant comme le bien y est plus auttentique» (p. 21). Christine passe ici de son propre jugement au jugement moins informé de lecteurs qui n'auront pas les mêmes raisons de peser et soupeser l'œuvre qu'elle. Ce qui l'amène à trancher l'oscillation de son jugement en faveur de la censure :

Mais je treuve, comme il me semble, ces dictes choses et asséz d'autres considerees, mieulx lui affiert ensevellissement de feu que couronne de lorier, non obstant que le claméz «mirouer de bien vivre, exemple de tous estas de soy politiquement gouverner et vivre religieusement et saigement » (p. 21).

«Ensevelissement de feu» fait allusion à l'enfer de tout le monde, alors que «couronne de laurier» fait allusion au paradis des écrivains. Pour Christine, les écrivains peuvent peut-être s'élever à un ordre de mérite hors du commun, mais ne peuvent pas se soustraire aux valeurs communes. Le Roman de la Rose ne peut pas être un «mirouer de bien vivre»s'il exclut les femmes de ce «bien vivre». La lettre ne se termine pas sur la condamnation du livre mais sur un défi à l'interlocuteur du débat: 
Et ne me soit imputé a follie, arrogance ou presompcion d'oser, moy femme, repprendre et redarguer aucteur tant subtil et son euvre admenuisier de louenge, quant lui, seul homme, osa entreprendre a diffamer et blasmer sans excepcion tout un sexe (p. 22).

La conversation civile entre hommes et femmes, le débat public entre gens de lettres des deux sexes est possible, dit Christine, si seulement vous me reconnaissez le droit, à moi femme, de répondre pour les femmes à celui qui en médit. Il suffisait de l'écrire et de quelques siècles de débat... mais ceci est une autre histoire.

Virginie Greene

Harvard University 